\title{
Shift in HIV/AIDS Epidemic in Southeastern China: A Longitudinal Study from 1987 to 2015
}

\author{
Yansheng Yan $1,2,3, *$, Shouli Wu ${ }^{1,2,3, *}$, Liang Chen ${ }^{1,2}$, Pingping Yan ${ }^{1,2}$, Yuefeng Qiu ${ }^{1}$, \\ Meirong Xie ${ }^{1}$, Zhenghua Wang ${ }^{1}$ and Xun Lin ${ }^{1}$ \\ 1 Fujian Provincial Center for Disease Control and Prevention, No. 76 Jintai Road, Fuzhou 350001, China; \\ fjcr@fjcdc.com.cn (L.C.); ypp13960789519@sina.com (P.Y.); best018@163.com (Y.Q.); \\ murongxie@hotmail.com (M.X.); hellomuscle@163.com (Z.W.); lx1031@163.com (X.L.) \\ 2 Fujian Provincial Key Laboratory of Zoonosis Research, No. 76 Jintai Road, Fuzhou 350001, China \\ 3 School of Public Health, Fujian Medical University, No. 88 Jiaotong Road, Fuzhou 350004, China \\ * Correspondence: yysh@fjcdc.com.cn (Y.Y.); shouli1975@sina.com (S.W.); \\ Tel.: +86-591-8752-5992 (Y.Y.); +86-591-8761-9263 (S.W.)
}

Academic Editor: Paul B. Tchounwou

Received: 13 June 2016; Accepted: 27 July 2016; Published: 6 August 2016

\begin{abstract}
Objective: The aim of this study was to investigate the shift in the epidemiological features of HIV / AIDS during the last three decades in Fujian Province, southeastern China, so as to provide evidence for the development of novel HIV/AIDS control strategies. Methods: Data pertaining to the conventional surveillance, sentinel surveillance and epidemiological survey in Fujian Province during the period from 1987 to 2015 were collected. The epidemiological trends were described, and the subtypes of HIV strain were genotyped. In addition, the response to antiretroviral therapy was evaluated, and HIV genotypic resistance was assayed. Results: There was an increasing trend observed in the reported cases of HIV/AIDS in Fujian Province. From 1987 to the end of 2015, a total of 8651 HIV / AIDS cases were reported across the province, with totally 1557 deaths found. Among the total cases, the ratio of male/female cases was 3.7:1, which appeared to be an increasing trend; $77.1 \%$ cases were detected in young and middle-aged populations aged 19 to 50 years, however, the new HIV infections recently tended to occur in young people aged 15 to 18 years and in populations aged 50 years and older. Among all infected individuals, $49.3 \%$ were married, however, the percentage of unmarried cases increased from $6.67 \%$ before 1994 to $40.1 \%$ in $2015 ; 64.8 \%$ had junior high school education or lower, however, the proportion of HIV / AIDS cases with junior college education or above gradually increased from $6.5 \%$ in 2009 to $21.4 \%$ in 2015 . The reported HIV / AIDS cases were predominantly found in coastal regions; however, a rapidly increasing trend was seen in the number of HIV/AIDS cases in inland regions, and the geographical variation of the cases gradually reduced. There were multiple routes of HIV transmission found in Fujian Province, and $94.2 \%$ infections were sexually transmitted, with a large increase in the percentage of male homosexual transmission. A variety of HIV-1 subtypes were genotyped in the province during the study period, and CRF01-AE and CRF07-BC intersubtype recombinant forms were predominant; however, a declining trend in the proportion of HIV-1 CRF01-AE recombinant virus and a significant rise in the proportion of HIV-1 CRF07-BC recombinant virus were observed. Over $90 \% \mathrm{HIV}$ inhibition was found in all cases receiving antiretroviral therapy during the period from 2011 to 2015, indicating a low prevalence of HIV drug resistance. Conclusions: An increasing trend is still observed in the HIV / AIDS epidemics in Fujian Province, southeastern China. However, the epidemiological pattern of HIV / AIDS has recently changed in the province, and effective control interventions targeting the shift in the epidemiological features of HIV / AIDS should therefore be implemented to control the spread of the epidemic.
\end{abstract}


Keywords: AIDS; HIV; spatial-temporal characteristics; antiretroviral therapy; molecular epidemiology; drug resistance; genotyping; Fujian Province

\section{Introduction}

Acquired immune deficiency syndrome (AIDS), caused by the fatal human immunodeficiency virus (HIV) infection, is not only a public health issue but also a social issue [1]. Since the first case of HIV infection was detected in the United States in 1981, the HIV / AIDS epidemic has spread across the world dramatically, and causes huge health, economic and social burdens [2]. In China, the first reported AIDS case was documented in an Argentine man travelling to China in 1985, and in the same year, the first local case with HIV infection was detected in a hemophilia patient in Zhejiang Province, eastern China [3]. Since then, a nation-wide spread of HIV/AIDS has been observed in the country [4], and till 2015, the HIV / AIDS epidemic has been reported in 31 provinces of China, with 577,423 people living with HIV / AIDS and 182,882 deaths [5].

Since the first HIV / AIDS case was reported in Fujian Province, southeastern China in 1987, an increasing trend has been seen in the HIV / AIDS epidemic in the province yearly, and a big change is found in the HIV/AIDS epidemic during the last three decades [6]. Overall, the transmission of HIV/AIDS seems to be classified into three phases based on the epidemic. In the first phase (from 1987 to 1994), the HIV / AIDS cases detected were predominantly imported from southeastern Asian countries, with few cases reported, and minor local cases were found to be infected through couple sexual behaviors; in the second phase (from 1995 to 2001), HIV infection was detected in local residents with sexual promiscuity, and a clear-cut increase in the number of HIV / AIDS patients and a remarkable speeding of the spread was observed, however, the overall epidemic appeared at a low level; and in the third phase (from 2002 to present), various routes of HIV transmission were observed, the infection spread from high risk population to general population, the number of infected cases increased significantly yearly, and the overall prevalence shifted from a low to moderate level $[7,8]$. Since the early 21st century, the HIV / AIDS epidemic has actually experienced great changes in the province due to the alteration of economic status, lifestyle and education [9]. The current study was therefore designed with a major aim to investigate the shifting pattern of the HIV / AIDS epidemic in Fujian Province, a southeastern coastal region of China, during a 29-year period from 1987 to 2015, to provide scientific evidence for revising the current strategy and developing new interventions for HIV / AIDS prevention and control.

\section{Materials and Methods}

\subsection{Data Source}

All data pertaining to the HIV / AIDS epidemic were captured from the National Data Information System for Comprehensive HIV / AIDS Control, National HIV Sentinel Surveillance System, and the National Information Management System of Antiretroviral Therapy of HIV/AIDS.

\subsection{HIV Detection}

Serum anti-HIV antibody was screened using an enzyme-linked immunosorbent assay (ELISA) or other rapid test kits, and those positive for anti-HIV antibody were validated using Western blotting assay [10].

\subsection{Monitoring of the Response to Antiretroviral Therapy}

The eligible HIV/AIDS patients that met the inclusion criteria of China's National Free Antiretroviral Treatment Program were given free antiretroviral therapy [11]. The HIV viral load was quantified using a Versant ${ }^{\circledR}$ HIV-1 RNA 3.0 Assay (bDNA; Bayer HealthCare; Tarrytown, 
NY, USA) on a Bayer Versant ${ }^{\circledR} 340$ bDNA Analyzer (Bayer HealthCare; Tarrytown, NY, USA) following the manufacturer's instructions, and the $\mathrm{CD}^{+} \mathrm{T}$ count was measured with a $\mathrm{BD}$ TriTEST ${ }^{\mathrm{TM}}$ CD3-FITC/CD4-PE/CD45-PerCP kit (BD Biosciences; Franklin Lakes, NJ, USA) on a BD FACSCalibur system (BD Biosciences; Franklin Lakes, NJ, USA).

\subsection{HIV Genotypic Resistance Testing}

The genotypic test for HIV drug resistance was performed using an in-house nested RT-PCR assay [12]. Briefly, the HIV-1 pol gene (protease 1-99 amino acids and part of the reverse transcriptase 1-252 amino acids) was amplified using the nested RT-PCR assay, and the purified PCR products were sequenced by the Sangon Biotech (Shanghai) Co., Ltd. (Shanghai, China). Then, the sequences were analyzed in the Stanford HIV Drug Resistance Database (http://hivdb.stanford.edu/), and the resistance level was classified into low-, intermediate- and high-level resistance based on mutation results.

\subsection{Identification of HIV Subtypes}

For the identification of HIV subtypes, the pol, gag and env genes of HIV were amplified using a nested RT-PCR assay [13], and sequenced. The sequences were edited and corrected in the software Vector NTI version 8.0, and aligned to the sequence of HIV-1 reference isolate in the software Bioedit version 7.0.1. Following format transformation in the software Mega version 4.0, phylogenetic tree was constructed using the neighbor-joining (NJ) algorithm. The genotype was initially identified using the BLAST and RIP programs in the Los Alamos National Laboratory of HIV Databases (http:/ / www.hiv.lanl.gov), and validated based on the phylogenetic tree analysis. Finally, the genotypes of the pol, gag and env gene regions were identified.

\subsection{Ethical Statement}

This study was approved by the Ethical Review Committee of Fujian Center for Disease Control and Prevention, China (permission No. 20150011). All experiments performed in this study were in accordance with the Declaration of Helsinki and national laws and regulations of China.

\subsection{Statistical Analysis}

A descriptive epidemiology was employed in this study. All data were double entered into Microsoft Excel 2007 (Microsoft; Redmond, WA, USA) and all statistical analyses were performed using the statistical software SPSS version 13.0 (SPSS Inc.; Chicago, IL, USA).

\section{Results}

\subsection{Temporal Shift of HIV/AIDS Patients}

During the period from 1987 to 2015, there was an increasing trend seen in the number of HIV-infected and AIDS patients in Fujian Province yearly, and the constituent ratio of subjects with AIDS status upon identification has gradually reduced. Recently, the expansion in the coverage of HIV voluntary counseling and testing (VCT) and the implementation of national expanded HIV testing initiative have resulted in a growth in the number of detected HIV infections, and the detection of HIV infection (approximately 2.5 million) in 2015 exceeded the sum of 20-year detection from 1987 to 2006 (approximately 2.3 million). Consequently, a sharp increase was found in the number of reported HIV / AIDS subjects, and there were 8651 accumulated HIV / AIDS cases reported in Fujian Province until 31 December 2015, with 1557 accumulated deaths reported. Currently, there are 7094 people living with HIV / AIDS, including 4347 HIV-infected cases and 2747 AIDS cases. In 2015, there were 2184 new HIV / AIDS cases (1627 HIV-infected cases and 557 AIDS cases), and 226 new deaths were reported, which increased by $21.9 \%$ and $0 \%$ relative to those in 2014 . In addition, the constituent ratio of subjects at AIDS status upon identification increased from 33.3\% in 2002 to $74.4 \%$ in 2015 (Figure 1). 


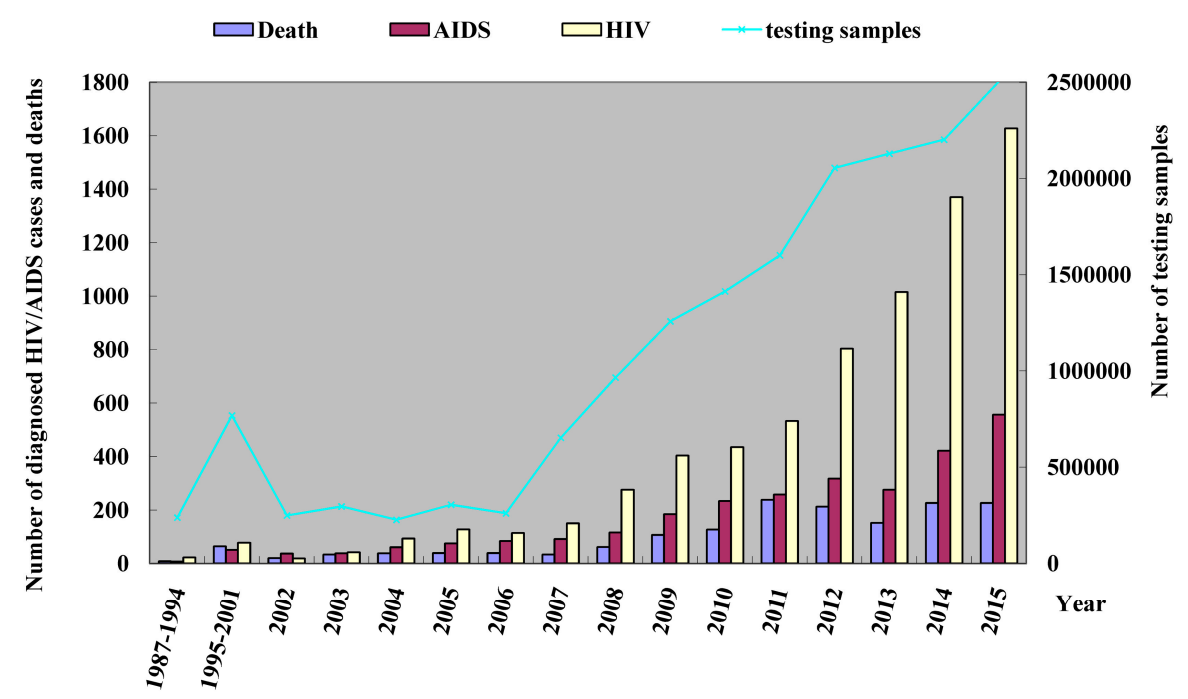

Figure 1. Temporal distribution of HIV / AIDS patients reported in Fujian Province from 1987 to 2015.

\subsection{Demographic Profile of HIV/AIDS Patients}

Among the HIV / AIDS cases reported in Fujian Province from 1987 to 2015, 78.9\% were male and $21.1 \%$ were female, with a male/female ratio of 3.7:1. Recently, the ratio of male to female cases appears to be increasing, from 1.8:1 in 2009 to 5.1:1 in 2015 (Figure 2).

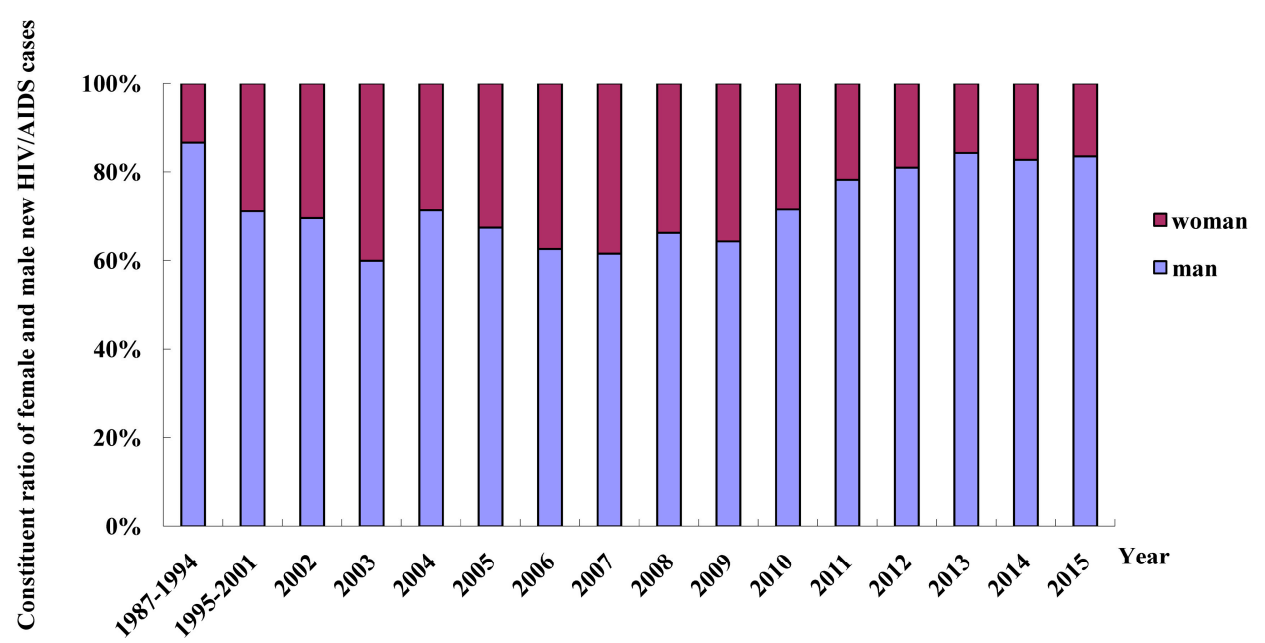

Figure 2. Gender-specific distribution of new HIV/AIDS patients reported in Fujian Province from 1987 to 2015.

The reported HIV / AIDS cases were predominantly found in young and middle-aged people, among which $77.1 \%$ were aged between 19 and 50 years. The cases had a mean age of $38.3 \pm 14.7$ years (range: nine months to 90 years), and the HIV-infected cases have recently tended to be detected in young and older populations. Since 2011, a remarkable rise has been seen in the number of HIV / AIDS cases in those aged 15 to 18 years, with a mean annual rise of $54 \%$ relative to that in 2010 . In addition, the percentage of HIV-infected cases aged 50 years and older has increased from $7.1 \%$ in 2002 to $25.7 \%$ in 2015 (Figure 3). 


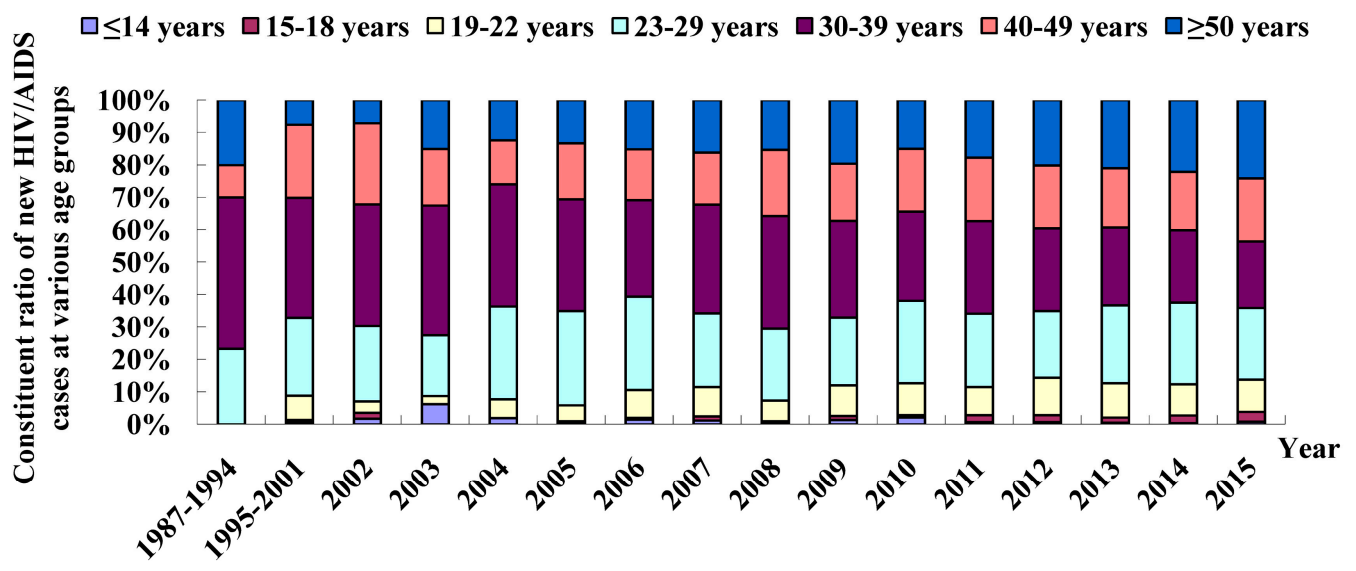

Figure 3. Age-specific distribution of new HIV/AIDS patients reported in Fujian Province from 1987 to 2015.

The HIV / AIDS cases were predominantly married; however, the proportion of unmarried cases has recently shown an increasing trend. Of the total reported HIV/AIDS cases, 4268 cases (49.3\%) were married, 2988 cases (34.5\%) were unmarried, 1165 cases (13.5\%) were divorced or widowed, and the remaining 230 cases $(2.7 \%)$ had unknown marital status. The percentage of unmarried cases was found to have increased from $6.67 \%$ before 1994 to $40.1 \%$ in 2015 (Figure 4).

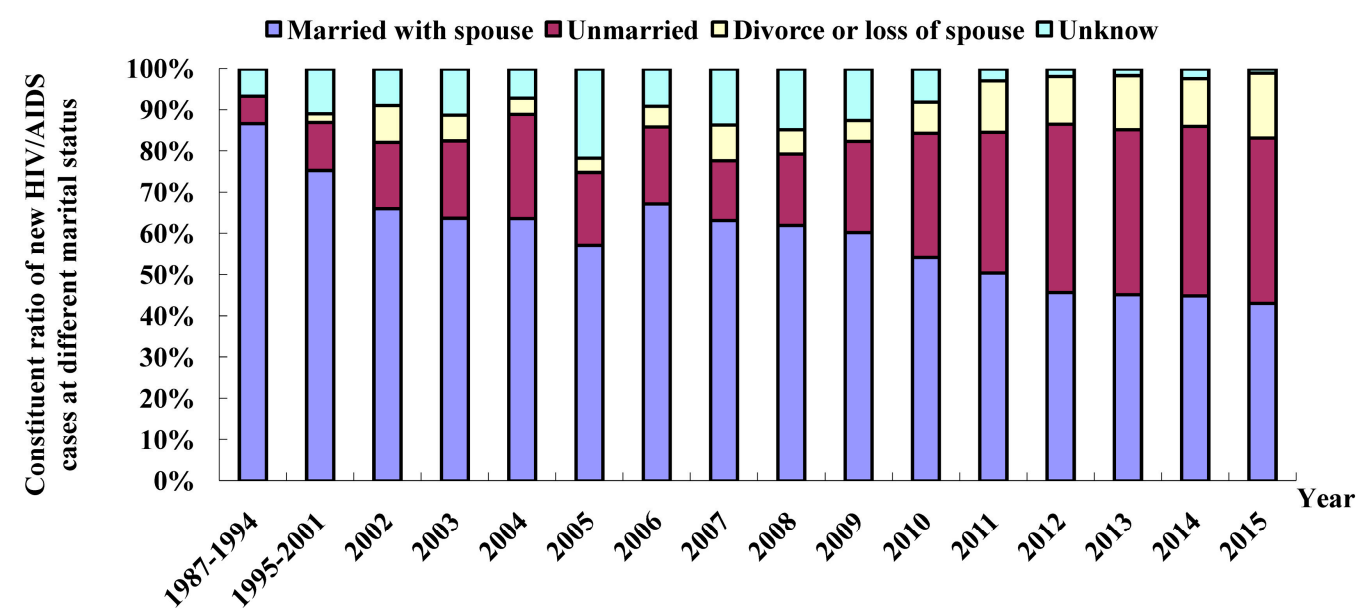

Figure 4. Distribution of new HIV/AIDS patients by marital status in Fujian Province from 1987 to 2015 .

The education levels of the HIV/AIDS cases were predominantly at a junior high school level or lower; however, the percentage of cases with junior college education or higher has appeared to rise. Of the total HIV / AIDS cases reported in Fujian Province from 1987 to 2015, there were 602 cases $(7.0 \%)$ with illiteracy level, 1998 cases $(23.1 \%)$ with primary school level, 3001 cases $(34.7 \%)$ with junior high school level, 1542 cases (17.8\%) with senior high school/special secondary school level, 1451 cases $(16.8 \%)$ with junior college level, and 57 cases $(0.7 \%)$ with unknown education level. In the last five years, the percentage of cases with junior college education or higher increased from $6.5 \%$ in 2009 to $21.4 \%$ in 2015 (Figure 5). 


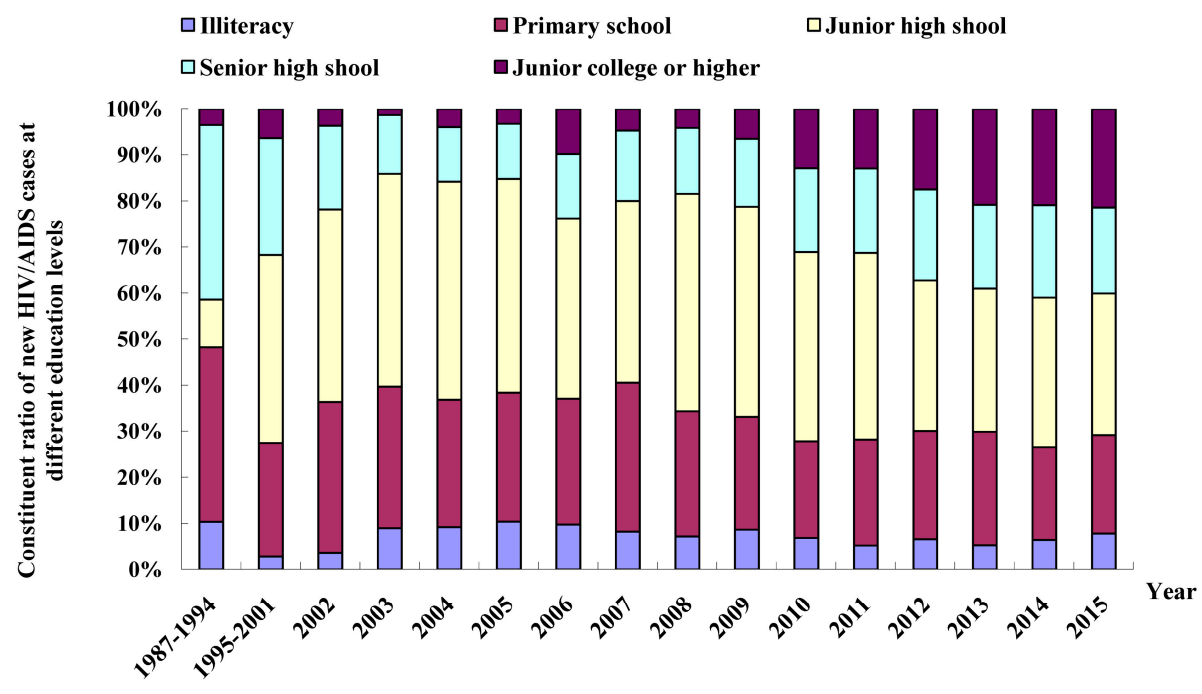

Figure 5. Distribution of new HIV/AIDS patients by education level in Fujian Province from 1987 to 2015.

\subsection{Geographic Features}

Since the first AIDS case was reported in Fujian Province in 1987, the HIV / AIDS patients have gradually spread from the coastal regions to inland regions, and there have been HIV/AIDS cases reported across the nine cities of the province since 2001. The reported HIV/AIDS cases were predominantly found in the coastal regions; however, a sharp increase was observed in the number of HIV / AIDS cases reported in the inland regions, and a gradually reduced regional variation was seen. Fuzhou (2317 cases), Quanzhou (1824 cases) and Xiamen (952 cases) cities were the top three prefectures that had the largest number of accumulated HIV/AIDS cases in the province, which consisted of 58.9\% (5093/8651) of the accumulated cases reported in Fujian Province from 1987 to 2015. In addition, Quanzhou (507 cases), Fuzhou (471 cases) and Xiamen (319 cases) cities ranked as the top three prefectures in the province with the largest number of new HIV/AIDS cases diagnosed in 2015, which consisted of 59.4\% (1297/2184) of all new cases reported in the province (Figure 6).

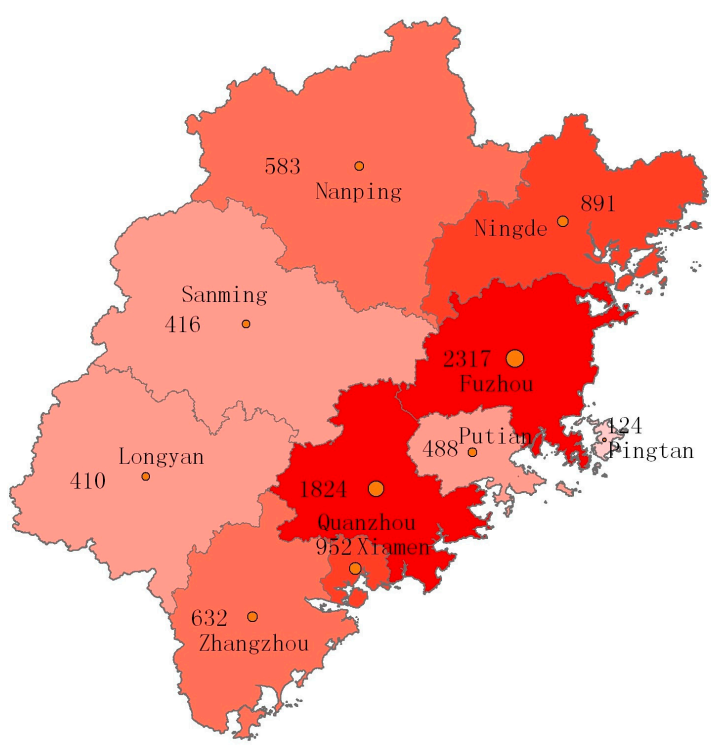

Figure 6. Geographic distribution of reported HIV / AIDS patients in Fujian Province from 1987 to 2015. 


\subsection{Routes of HIV Infection in Fujian Province}

A variety of routes of HIV infection were found in Fujian Province, in which sexual transmission was predominant and its constituent ratio increased, with a large rise seen in the percentage of male homosexual transmission. Of the accumulated HIV / AIDS cases reported in Fujian Province from 1987 to 2015,6150 cases $(71.1 \%)$ were infected through heterosexual transmission, 1998 cases $(23.1 \%)$ through homosexual transmission, 164 cases $(1.9 \%)$ through drug injection, nine cases $(0.1 \%)$ through blood/plasma collection, 47 cases $(0.5 \%)$ through blood/blood product transfusion, 72 cases $(0.8 \%)$ through mother-to-child transmission, 17 cases $(0.2 \%)$ through sexual contact + drug injection, and 192 cases (2.2\%) with unknown transmission routes (Figure 7). Among the new HIV/AIDS cases diagnosed per year, the proportion of sexual transmission increased from $47.4 \%$ in 2004 to $97.4 \%$ in 2015, which mainly is attributed to the large increase in the percentage of male homosexual transmission. Since the first HIV/AIDS case through male homosexual transmission $(0.5 \%)$ was reported in Fujian Province in 2005, the proportion of male homosexual transmission has increased yearly, and reached $31.4 \%$ in 2015. Since the first HIV / AIDS case through mother-to-child transmission was documented in 2002, few cases have been reported each year except 2005. In 2003, the first case with HIV infection via drug use was detected in a local resident, and since then, a low prevalence of HIV infection has been observed in drug users in the province (Figure 8).
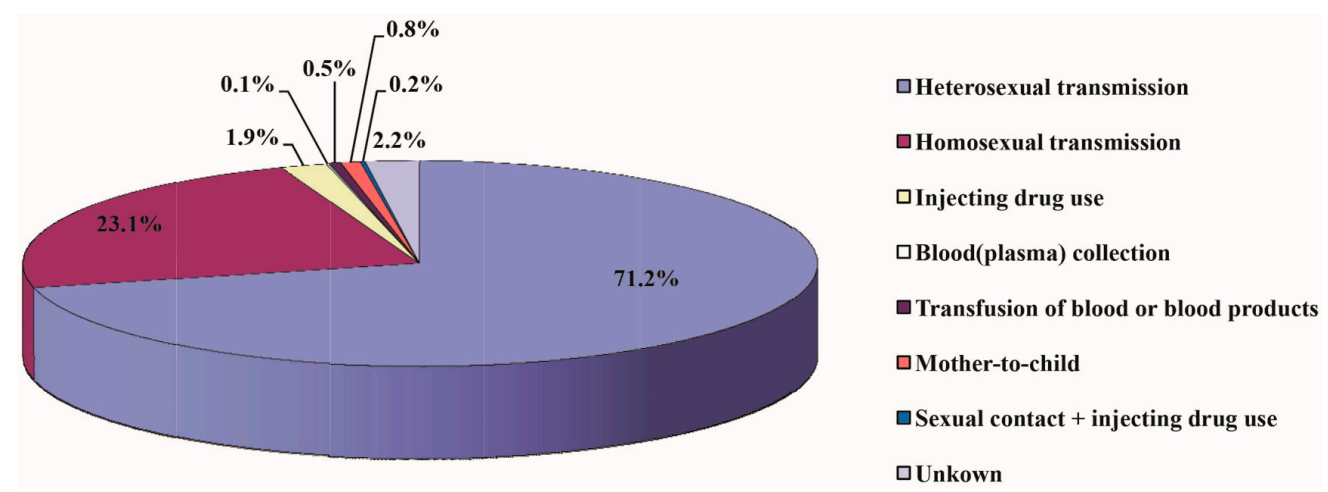

Figure 7. Routes of transmission of all reported HIV / AIDS cases in Fujian Province from 1987 to 2015.

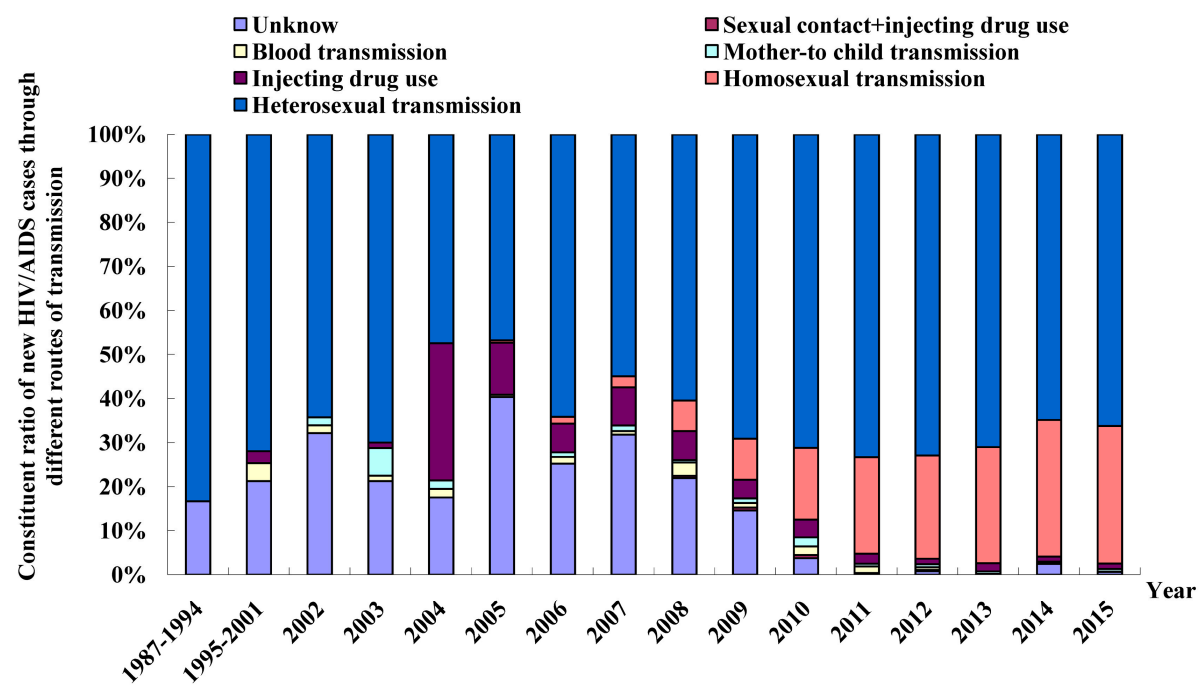

Figure 8. Routes of transmission of new HIV/AIDS cases in Fujian Province from 1987 to 2015. 


\subsection{HIV Sentinel Surveillance}

A total of 39 HIV / AIDS sentinel surveillance sites were assigned in Fujian Province during the period from 2010 to 2015, which covered men who have sex with men (MSM), commercial sex workers, long-distance male bus drivers, mobile population, male patients attending STD clinics, young students, drug users and pregnant women, and various changing trends were seen in the positive rate of anti-HIV antibody among different subjects. There was a large rise in the positive rate of anti-HIV antibody in MSM, and a more remarkable increase $(16.26 \%)$ was notably observed in 2015, with an over two-fold greater increase (7.05\%) in 2010. In addition, a low prevalence of anti-HIV antibody was detected, except a slight rise in subjects with STD (the highest prevalence was $0.77 \%$ ), notably in pregnant women/puerperae, who were negative for anti-HIV antibody during the monitoring period from 2010 to 2015 except in 2012 when a pregnant woman was positive for anti-HIV antibody (Figure 9).

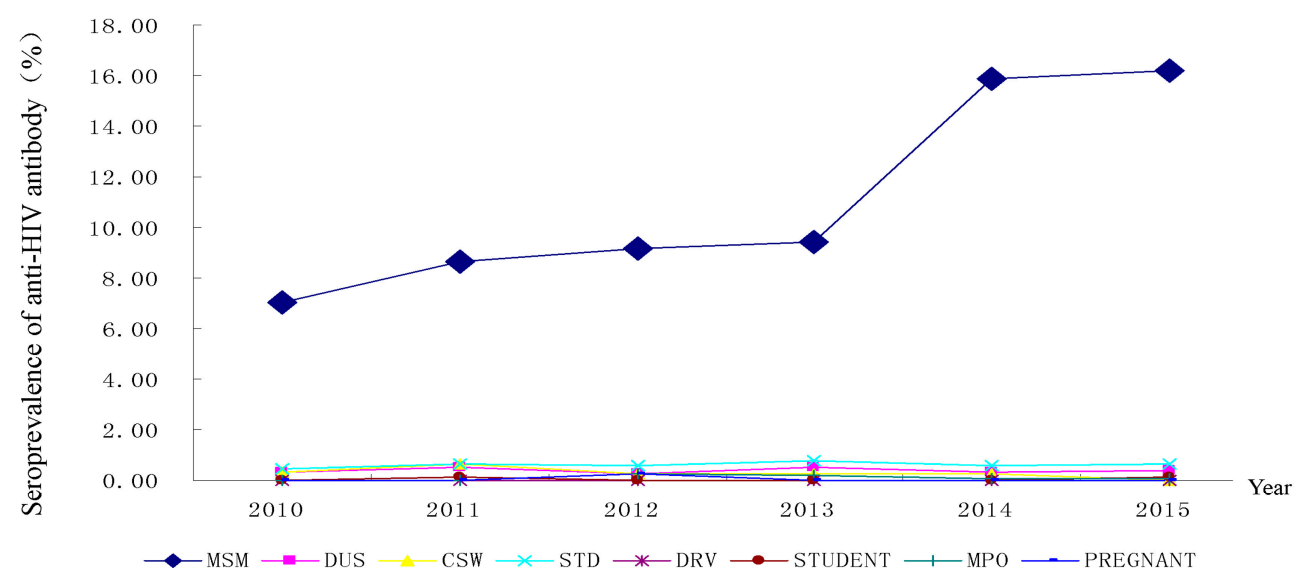

Figure 9. Seroprevalence of anti-HIV antibody in sentinel sites of Fujian Province from 2010 to 2015. MSM, men who have sex with men; DUS, drug users; CSW, commercial sex workers; STD, male patients attending STD clinics; DRV, long-distance male bus drivers; STUDENT, young students; MPO, mobile population; PREGNANT, pregnant women.

\subsection{Shift in HIV-1 Subtypes}

To investigate the distribution and shift of HIV-1 subtypes in Fujian Province, four molecular epidemiological surveys of HIV have been carried out in Fujian Province from 2000 to 2012 [14-16]. A variety of HIV-1 subtypes were determined in Fujian Province, in which CRF01-AE recombinant subtype was predominant and A, B, and C subtypes and BC, 12B, CRF55-01B and CRF68-01B intersubtype recombinant forms were also detected. In addition, other suspected new HIV-1 intersubtype recombinant viruses, predominantly recombinant forms of CRF01-AE and B or BC, were found. There was a shift in the distribution of HIV-1 subtypes at various study periods, and the constituent ratios of CRF01-AE intersubtypes and B subtypes appeared to have a gradual declining trend yearly, decreasing from $75.6 \%$ and $17.1 \%$ in 2001 to $46.9 \%$ and $6.17 \%$ in 2012 , respectively. Since HIV-1 BC intersubtype recombinant form was first reported in Fujian Province in 2002, the proportion of this virus has shown a remarkable rise, with an increase from $14.3 \%$ in 2003 to $42 \%$ in 2012, in which CEF07-BC form was predominant (Figure 10).

Recently, a sharp increasing trend has been found in the number of HIV infections among MSM. Sequencing analysis of the HIV-1 strains isolated from the infected MSM showed that CRF01-AE, CRF07-BC and B subtypes were predominant, which was the same with the HIV-1 subtypes in other subjects in the province, and the proportion of CRF01-AE and B subtypes showed a declining trend, while the percentage of CRF07-BC subtype increased; in addition, new HIV-1 subtypes of 
CRF5501-B and CRF68-01B and suspected new recombinant forms (predominantly recombinant forms of CRF01-AE and B or BC) were detected (Figure 11).

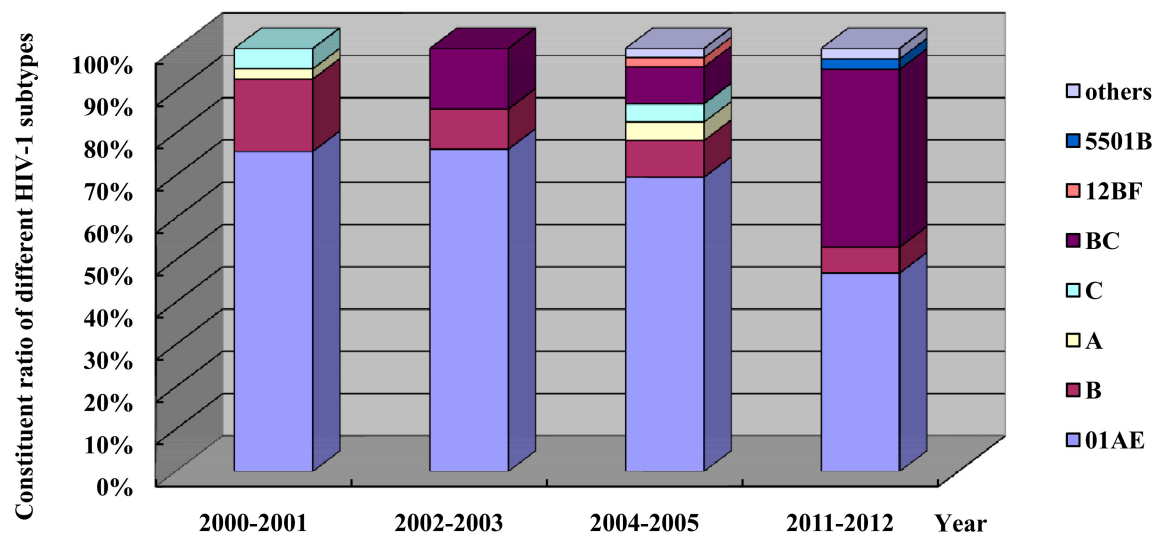

Figure 10. Distribution and shift of HIV-1 subtypes in Fujian Province, revealed by four molecular epidemiological surveys of HIV.

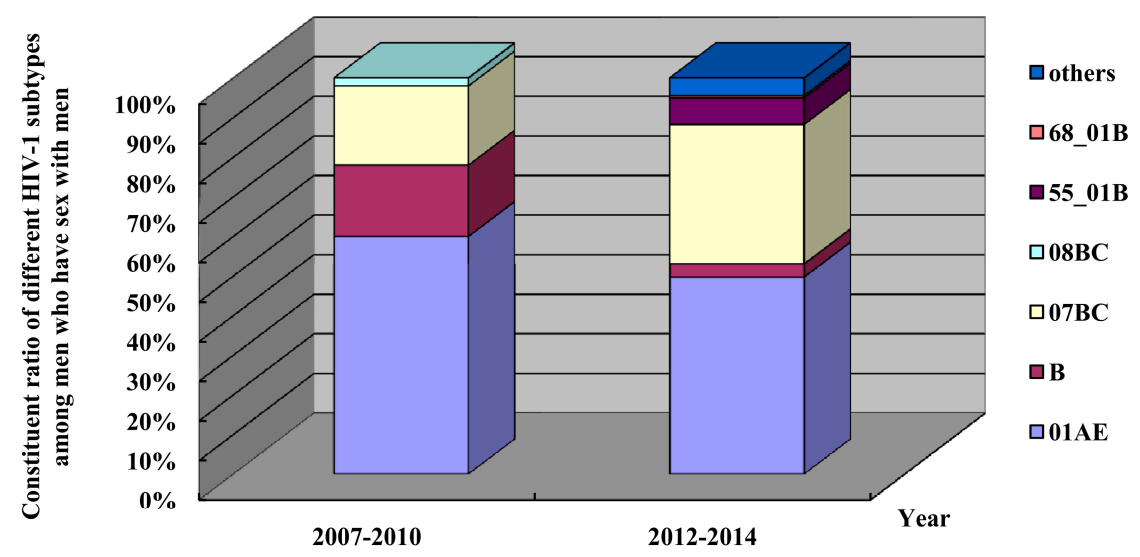

Figure 11. Distribution and shift of HIV-1 subtypes among men who have sex with men in Fujian Province, revealed by four molecular epidemiological surveys of HIV.

\subsection{Effectiveness of Antiretroviral Treatment}

In 2003, the "Four-Free-One-Care" policy was introduced, which provided free antiretroviral treatment for all eligible HIV patients in China [17], and the free antiretroviral treatment program was initiated in Fujian Province in 2005. Currently, there are 40 designated medical institutions undertaking free antiretroviral therapy for HIV/AIDS across the province. With the implementation of the coverage of expanded HIV / AIDS therapy, the number of HIV/AIDS cases receiving antiretroviral therapy has increased by $34 \%$ to $47 \%$ annually during the latest five years. Until the end of 2015 , a total of 5808 HIV/AIDS cases have been given free antiretroviral therapy. There are currently 5041 cases undergoing treatment, including 5001 adults and 40 children. Periodical monitoring of HIV viral load and drug resistance showed a rising trend in the inhibition of HIV (viral load < 1000 copies $/ \mathrm{mL}$ ), and over $90 \%$ inhibition was seen in the last five years, with the highest inhibition seen being $95.2 \%$ (Figure 12). The patients with HIV virological failure (viral load $>1000$ copies $/ \mathrm{mL}$ ) were subject to genotypic drug resistance testing, and the gene sequences of proteases and some reverse transcriptases were obtained from the sequencing data of 275 samples. Drug resistance testing showed a low overall prevalence of HIV-1 drug resistance in Fujian Province, and a total of 145 cases with failure in antiretroviral therapy developed resistance to reverse transcriptase and protease inhibitor (PI) at various levels, with $39.27 \%$ (108/275), 51.64\% (142/275) and 2.55\% (7/275) prevalence of resistance 
to nucleoside reverse transcriptase inhibitor (NRTI), non-nucleoside reverse transcriptase inhibitor (NNRTI) and PIs, respectively (Figure 13). In addition, periodical monitoring of primary HIV drug resistance in treatment-naïve subjects showed a low overall prevalence of primary drug resistance in treatment-naïve subjects; however, a rise was seen in the prevalence of primary drug resistance in treatment-naïve HIV-infected MSM, including eight cases (4.47\%) with potential resistance of PI, four cases $(2.23 \%)$ with potential or mild-level resistance to NRTI and 10 cases $(5.58 \%)$ with resistance to NNRTI at more than mild levels.

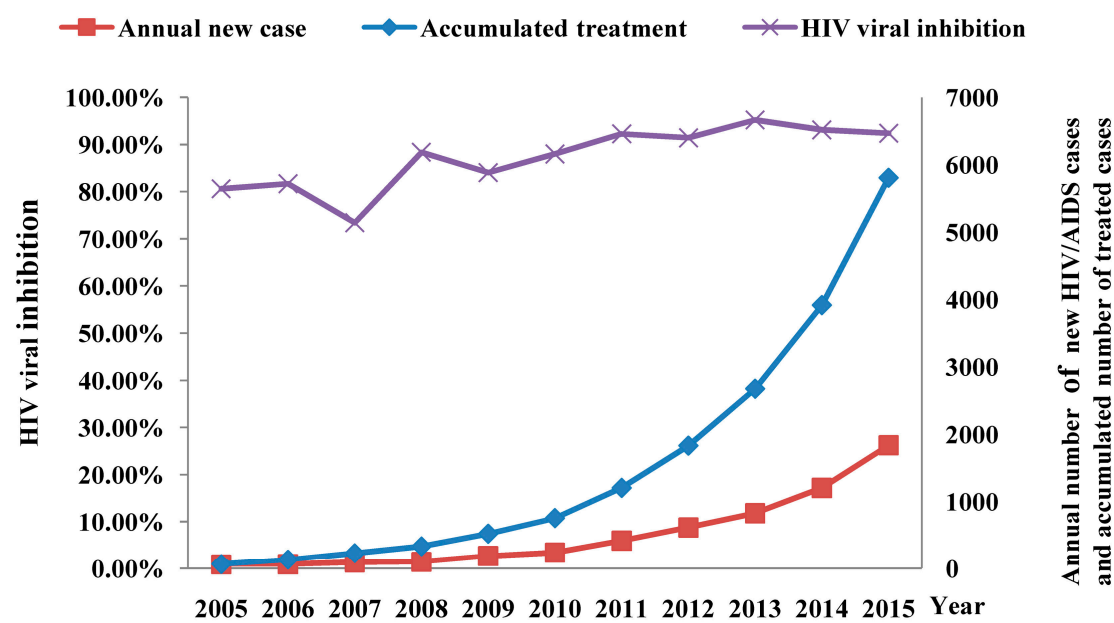

Figure 12. Effectiveness of free antiretroviral treatment program in Fujian Province from 2005 to 2015.

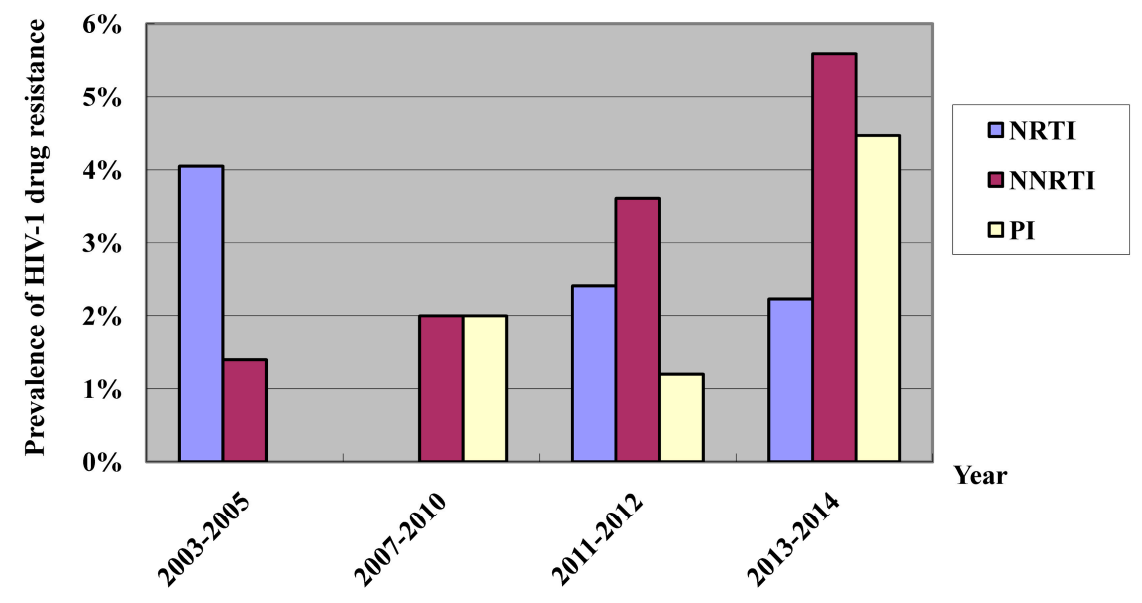

Figure 13. Primary HIV drug resistance in antiretroviral therapy-naive HIV/AIDS patients in Fujian Province from 2003 to 2014.

\section{Discussion}

During the last 30 years, there has been a shift in the epidemiological features of HIV / AIDS in Fujian Province. In general, the following characteristics were seen: (1) There was an increasing trend observed in the reported cases of HIV/AIDS in Fujian Province, and the spread of HIV / AIDS epidemic accelerated; however, a low mortality of HIV/AIDS patients was maintained; (2) Young and middle-aged populations remain the predominant subjects affected by HIV / AIDS; however, HIV infections have recently tended to be found in 15 to 18 year olds and the elderly (50 years and older). In addition, the infected subjects were predominantly male, and the male/female ratio appears to be increasing, while a rise was also seen in the proportion of unmarried people; (3) Multiple routes of HIV transmission were found, in which sexual transmission was predominantly observed, and its constituent ratio continued to rise. The proportion of male homosexual transmission increased greatly, 
while a low percentage of HIV transmission via injecting drug use was present during the whole period; (4) The HIV / AIDS epidemic has spread across the province, and a gradually reduced regional variation was seen. Although the currently reported HIV/AIDS cases were predominantly found in coastal regions, a rapidly increasing trend has been found in the number of cases in the inland regions; (5) A variety of HIV-1 subtypes were detected, in which CRF01-AE and BC recombinant forms were predominantly found, the constituent ratio of CRF01-AE and B subtypes gradually reduced yearly, the $\mathrm{BC}$ recombinant form increased remarkably and new intersubtype recombinant viruses were continuously detected; (6) The coverage of antiretroviral therapy continued to expand, satisfactory effectiveness was achieved, and a low overall prevalence of HIV drug resistance was detected.

Unsafe sexual behavior has been identified as the major cause of HIV infection [18,19]. In China, the lack of sex education in schools may be one of the causes responsible for the transmission of HIV/AIDS among younger populations, and parents seldom give sex-related education and interventions to their children because of Chinese traditional values. Recently, there has been a remarkable rise in the number of MSM among young populations, and the presence of social apps has lead to easier development of sexual behaviors between MSM, among whom the majorities have unsafe sexual behaviors without using condoms. In addition, these MSM may select to marry women due to social and family pressure, resulting in the spread of HIV infections from high-risk populations to general populations. Recently, the health status of elderly people has gradually improved with an increase in sexual demand, and these people have spare time and a high economic status. In addition, empty nests, loss of spouses, and reduced sexual function promote the pursuit for sexual stimulus, and as they lack awareness of HIV / AIDS prevention, during commercial sexual behaviors, there is a low use of condoms. Moreover, the sexual behaviors mainly occur in rented houses, street-side salons and small inns, thereby resulting in an increase in the risk of exposure to HIV infection. Therefore, targeted interventions should be implemented, such as strengthening of sex education at schools, establishment of school HIV/AIDS epidemic informing system and improvement of interventions to prostitutes, and health education and consultations pertaining to HIV / AIDS prevention and control should be given to elderly people, as well as much attention being paid to adolescents with HIV / AIDS, to prevent and control the further spread and transmission of HIV / AIDS in elderly populations.

Since the first case of HIV infection was reported in local drug users in Fujian Province in 2003, a low prevalence of HIV infection has been detected in drug users in the province, which may be associated with the low use of sharing needles and the effective implementation of methadone treatment. However, the threat of club drugs misuse to the transmission of HIV cannot be neglected. Relative to conventional opioid drugs, club drugs mainly refer to artificial, chemically synthesized or semi-synthesized substances like amphetamines, ketamines and triazolam [20]. These chemicals do not transmit HIV-1 virus through shared use of injectors, however, they may cause cognitive disorders and an increase in unsafe sexual behaviors among drug users, which consequently leads to the emergence of complicated sexual partner networks and sexual behaviors patterns, thereby resulting in an increase in the risk of HIV infection via sexual transmission [21]. Therefore, the effect of club drugs misuse on the transmission of HIV should be paid more attention [22].

Since the first case of AIDS was detected in Fujian Province in 1987, a remarkable shift has been observed in the HIV-1 subtypes in the province. The proportion of CRF01-AE and B subtypes has gradually reduced yearly, and the intersubtype BC recombinant form significantly has increased, with novel subtypes continuously identified. The HIV-1 CRF01-AE subtype was firstly identified in female sex workers (FSWs) and injection drug users (IDUs) in Yunnan and Guangxi, China in 1994 to 1996; however, this subtype has spread across the country, and has become widespread among the HIV-infected populations through heterosexual transmission, which has become the predominant epidemic subtype in Fujian Province [23-25]. Since the China-specific HIV-1 intersubtype B'/C recombinant forms (HIV/AIDS cases with CRF07-BC and CRF08-BC recombinant viruses) were first reported in Fujian Province in 2002 and 2003 [16], the CRF07-BC virus has gradually expanded, and has already become the second most predominant recombinant virus in the province, with a rising trend 
seen. With the widespread transmission of HIV CRF01-AE and CRF07-BC subtypes, the recombinant HIV strains carrying these two subtypes have already emerged in HIV-infected cases in Fujian Province. Therefore, the continuous expansion of high-risk population and communication frequency enables an increase in the possibility of HIV strain variation and recombination, resulting in emergence, spread and transmission of new HIV-1 strains, which challenges the diagnosis and molecular epidemiology of HIV / AIDS in Fujian Province, and proposes new problems of HIV / AIDS prevention and control that are urgently needed to be solved in China [26].

\section{Conclusions}

Currently, a preliminary outcome of antiretroviral therapy has been achieved and the mortality of AIDS has been remarkably reduced in Fujian Province [27]. However, the continuous expansion of the coverage of antiretroviral therapy and extension of treatment time will undoubtedly result in resistance to first-line antiretroviral therapy among more and more HIV / AIDS patients [28-30]. Monitoring of HIV drug resistance in treatment-naïve subjects, notably newly diagnoses HIV-infected subjects, is of great significance for the identification of drug-resistant isolates and to evaluate the likelihood of transmission of drug-resistant isolates. Periodical monitoring revealed a low overall prevalence of primary drug resistance in treatment-naïve subjects in Fujian Province; however, a rise has been seen recently. Therefore, we should strengthen the monitoring of HIV drug resistance and periodically assess the effectiveness of the currently used treatment regimens to provide scientific guidance for the selection of novel antiretroviral therapy regimens in drug-resistant HIV / AIDS cases. In addition, the adherence to antiretroviral therapy should be improved to enable the effectiveness of antiretroviral therapy and prevent the emergence and spread of multiple drug resistance (MDR) and cross resistance.

Acknowledgments: The authors would like to thank all participants from the Institute of STD and AIDS, Fujian Provincial Center for Disease Control and Prevention for their participation in this project. This study was supported by the grants from the Cultivation of Young Talents Project Fund from the Health Department of Fujian Province (grant No. 2015-ZQN-ZD-11), the Pilot Project of Fujian Provincial Department of Science \& Technology (grant no. 2016Y0010) and Fujian Provincial Medical Innovative Project (grant No. 2012-CX-12).

Author Contributions: Yansheng Yan and Shouli Wu conceived and designed the experiments; Shouli Wu, Liang Chen, Pingping Yan, Yuefeng Qiu, Meirong Xie, Zhenghua Wang and Xun Lin performed the experiments; Shouli $\mathrm{Wu}$ collected and analyzed the data; Shouli $\mathrm{Wu}$ prepared the first version of the manuscript; and Yansheng Yan revised and finalized the manuscript. All authors read and approved the final version of the manuscript.

Conflicts of Interest: The authors declare no conflict of interest.

\section{References}

1. Hogg, R.; Cahn, P.; Katabira, E.T.; Lange, J.; Samuel, N.M.; O'Shaughnessy, M.; Vella, S.; Wainberg, M.A.; Montaner, J. Time to act: Global apathy towards HIV/AIDS is a crime against humanity. Lancet 2002, 360, 1710-1711. [PubMed]

2. Walker, N.; Grassly, N.C.; Garnett, G.P.; Stanecki, K.A.; Ghys, P.D. Estimating the global burden of HIV/AIDS: What do we really know about the HIV pandemic? Lancet 2004, 363, 2180-2185. [CrossRef]

3. Wu, Z.; Rou, K.; Cui, H. The HIV/AIDS epidemic in China: History, current strategies and future challenges. AIDS Educ. Prev. 2004, 16, 7-17. [CrossRef] [PubMed]

4. Sheng, L.; Cao, W.K. HIV/AIDS epidemiology and prevention in China. Chin. Med. J. (Engl.) 2008, 121, 1230-1236. [PubMed]

5. National Center for AIDS/STD Control and Prevention. Update on the AIDS/STD epidemic in China and main response in control and prevention in December 2015. Chin. J. AIDS/STD 2016, 22, 69. (In Chinese)

6. Xia, P.; Okumura, J.; Yan, P.; Xie, M.; Wu, S.; Zhuang, M.; Zheng, J.; Zhang, C.; Zhang, M.; Kato, M.; et al. Steps towards preventive HIV treatment in Fujian, China: Problems identified via an assessment of initial antiretroviral therapy provision. PLOS ONE 2013. [CrossRef] [PubMed]

7. Yan, Y.; Zheng, Z.; Shao, Y. Analysis on the epidemic characterization and trend of AIDS in Fujian Province. Chin. J. Epidemiol. 1999, 20, 23-26. (In Chinese) 
8. Chen, K.; Yan, Y.S.; Chen, L.; Lin, X.; Yan, P.P.; Zheng, J. Analysis of HIV / AIDS prevalence features and trend in Fujian Province. China. J. AIDS/STD 2005, 11, 34-37. (In Chinese)

9. Wang, N. Some new trends of HIV/AIDS epidemic in China. Chin. J. Epidemiol. 2010, 31, 1205-1209. (In Chinese)

10. Dong, X.; Zhao, X.; Li, T.C.; Zhuang, T. Comparative analysis of the three editions of the national technical and working standards for AIDS testing. Chin. J. AIDS/STD 2010, 16, 595-598. (In Chinese)

11. Zhang, F.J.; Pan, J.; Yu, L.; Wen, Y.; Zhao, Y. Current progress of China's free ART program. Cell. Res. 2005, 15, 877-882. [CrossRef] [PubMed]

12. Chen, J.H.; Wong, K.H.; Chan, K.; Lam, H.Y.; Lee, S.S.; Li, P.; Lee, M.P.; Tsang, D.N.; Zheng, B.J.; Yuen, K.Y.; et al. Evaluation of an in-house genotyping resistance test for HIV-1 drug resistance interpretation and genotyping. J. Clin. Virol. 2007, 39, 125-131. [CrossRef] [PubMed]

13. Wei, M.; Guan, Q.; Liang, H.; Chen, J.; Chen, Z.; Hei, F.; Feng, Y.; Hong, K.; Huang, H.; Xing, H.; et al. Simple subtyping assay for human immunodeficiency virus type 1 subtypes B, C, CRF01-AE, CRF07-BC, and CRF08-BC. J. Clin. Microbiol. 2004, 42, 4261-4267. [CrossRef] [PubMed]

14. Yan, Y.S.; Weng, Y.W.; Chen, G.; Shao, Y.M.; Wang, H.R.; Pan, P.L.; Chen, L.; Wu, S.L.; Zheng, Z.S.; Zheng, J. Relationship between epidemiology of HIV-1 infection and HIV-1 subtypes in Fujian Province. Chin. J. Epidemiol. 2001, 22, 428-431. (In Chinese)

15. Deng, Y.Y.; Zhang, C.Y.; Yan, Y.S.; Yan, P.P.; Wu, S.L. Genetic subtype and epidemiological feature of HIV-1 circulating strains among recently infected patients in Fujian Province. Chin. J. Epidemiol. 2014, 35, $93-98$. (In Chinese)

16. Liu, J.F.; Yan, Y.S.; Lin, X.; Huang, H.L.; Yan, P.P.; Chen, G.; Zheng, J. Genetic subtype and epidemiological feature of HIV-1 circulating strains in Fujian Province. Chin. J. Zoonos. 2006, 22, 601-606. (In Chinese)

17. Lou, J.; Bu, L.C.; Han, E.; Ruan, Y.H.; Xing, H.; Shao, Y.M. Modeling primary and secondary drug resistances under China's "Four-Free-One-Care Policy". Int. J. Biomath. 2012. [CrossRef]

18. Wolf, K.; Young, J.; Rickenbach, M.; Vernazza, P.; Flepp, M.; Furrer, H.; Bernasconi, E.; Hirschel, B.; Telenti, A.; Weber, R.; et al. Prevalence of unsafe sexual behavior among HIV-infected individuals: The Swiss HIV Cohort Study. J. Acquir. Immune. Defic. Syndr. 2003, 33, 494-499. [CrossRef] [PubMed]

19. Glass, T.R.; Young, J.; Vernazza, P.L.; Rickenbach, M.; Weber, R.; Cavassini, M.; Hirschel, B.; Battegay, M.; Bucher, H.C. Is unsafe sexual behaviour increasing among HIV-infected individuals? AIDS 2004, 18, 1707-1714. [CrossRef] [PubMed]

20. Britt, G.C.; McCance-Katz, E.F. A brief overview of the clinical pharmacology of "club drugs". Subst. Use Misuse 2005, 40, 1189-1201. [CrossRef] [PubMed]

21. Parks, K.A.; Kennedy, C.L. Club drugs: Reasons for and consequences of use. J. Psychoact. Drugs 2004, 36, 295-302. [CrossRef] [PubMed]

22. Colfax, G.; Guzman, R. Club drugs and HIV infection: A review. Clin. Infect. Dis 2006, 42, $1463-1469$. [PubMed]

23. Feng, Y.; He, X.; Hsi, J.H.; Li, F.; Li, X.; Wang, Q.; Ruan, Y.; Xing, H.; Lam, T.T.; Pybus, O.G.; et al. The rapidly expanding CRF01_AE epidemic in China is driven by multiple lineages of HIV-1 viruses introduced in the 1990s. AIDS 2013, 27, 1793-1802. [CrossRef] [PubMed]

24. Su, Y.; Liu, H.; Wu, J.; Zhu, L.; Wang, N. Distribution of HIV-1 genotypes in China: A systematic review. Chin. J. Epidemiol. 2014, 35, 1164-1168. (In Chinese)

25. He, X.; Xing, H.; Ruan, Y.; Hong, K.; Cheng, C.; Hu, Y.; Xin, R.; Wei, J.; Feng, Y.; Hsi, J.H.; et al. A comprehensive mapping of HIV-1 genotypes in various risk groups and regions across China based on a nationwide molecular epidemiologic survey. PLOS ONE 2012. [CrossRef] [PubMed]

26. Wang, N.; Zhong, P. Molecular epidemiology of HIV in China: 1985-2015. Chin. J. Epidemiol. 2015, 36, 541-546. (In Chinese)

27. Wu, S.; Qiu, Y.; Yan, P.; Wang, W.; Xie, M.; Xia, P.; Wang, Z.; Yan, Y. Effectiveness of first-line antiretroviral therapy in HIV/AIDS patients: A 5-year longitudinal evaluation in Fujian Province, Southeast China. Arch. Virol. 2015, 160, 2693-2701. [CrossRef] [PubMed]

28. Liu, H.; Ma, Y.; Su, Y.; Smith, M.K.; Liu, Y.; Jin, Y.; Gu, H.; Wu, J.; Zhu, L.; Wang, N. Emerging trends of HIV drug resistance in Chinese HIV-infected patients receiving first-line highly active antiretroviral therapy: A systematic review and meta-analysis. Clin. Infect. Dis. 2014, 59, 1495-1502. [CrossRef] [PubMed] 
29. Wang, J.; Wang, Z.; Liu, J.; Yue, Y.; Yang, S.; Huang, H.; He, C.; Liao, L.; Xing, H.; Ruan, Y.; et al. Efficacy and HIV drug resistance profile of second-line ART among patients having received long-term first-line regimens in rural China. Sci. Rep. 2015, 5, 14823. [CrossRef] [PubMed]

30. Wang, X.; He, C.; Xing, H.; Liao, L.; Xu, X.; He, J.; Liu, Y.; Ling, H.; Liang, S.; Hsi, J.H.; et al. Emerging transmitted HIV type 1 drug resistance mutations among patients prior to start of first-line antiretroviral therapy in middle and low prevalence sites in China. AIDS Res. Hum. Retroviruses 2012, 28, 1637-1639. [CrossRef] [PubMed]

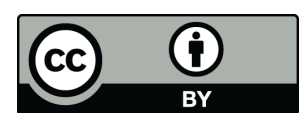

(C) 2016 by the authors; licensee MDPI, Basel, Switzerland. This article is an open access article distributed under the terms and conditions of the Creative Commons Attribution (CC-BY) license (http://creativecommons.org/licenses/by/4.0/). 